\title{
10 Fragen zum Schwerpunktthema „Pulmonale arterielle Hypertonie“
}

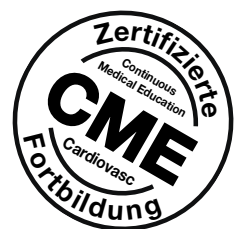

Die Multiple-Choice-Fragen rekrutieren sich aus dem Schwerpunktbeitrag von Ewert und Mitarbeitern (S. 44-51). Sowohl der Beitrag als auch die Fragen wurden im Hinblick auf Korrektheit und Relevanz für die Lesergruppe zweifach begutachtet. Die Antworten auf die CMEFragen ergeben sich nur zum Teil direkt aus dem Text; einige Fragen beruhen auf medizinischem Basiswissen! Es wird jeweils nur eine Antwort gesucht. Teilnahme nur im Internet unter www.cme-punkt.de. Dort führen wir ein elektronisches Punktekonto für Sie. Teilnahme-

schluss: 8.10. 2010.

II 1. Wer gilt als Erstbeschreiber des Rechtsherzkatheters beim Menschen?

A) Der französisch-amerikanische Kardiologe André Cournand

B) Der deutsche Chirurg Werner Forstmann

C) Der englische Kardiologe Paul Wood

D) Der englische Wissenschaftler Stephen Hales

E) Der deutsche Pathologe Rudolph Virchow

II 2. Welche Medikamente wurden als Erste erfolgreich zur spezifischen Behandlung von Patienten mit pulmonaler Hypertonie (PH) eingesetzt?

A) Diuretika und Sauerstoff

B) Vasodilatatoren wie Hydralazin oder Phentolamin

C) Kalziumkanal-Blocker

D) Prostazyklin i.v.

E) Orale Antikoagulazien

II 3. Wann wurde weltweit erstmals ein spezifisches Medikament zur Behandlung der primären pulmonalen Hypertonie (PPH) zugelassen?

A) $1973 \mathrm{im}$ Rahmen der ersten PPH-Weltkonferenz

B) nach den ersten positiven Studien mit Kalzium-Kanal-Blockern 1985

C) nach der ersten erfolgreichen Gabe von Epoprostenol 1979

D) nach der ersten randomisierten Studie mit Epoprostenol 1995

E) im Rahmen der zweiten PPH-Weltkonferenz 1998

II 4. Seit wann existiert eine internationale diagnostische Klassifikation der PH?

A) Seit der 1. PPH-Weltkonferenz 1973

B) Seit der 2. PPH-Weltkonferenz 1998 in Frankreich
C) Seit den Veröffentlichungen der amerikanischen Registerdaten 1987

D) Seit der Zulassung von Epoprostenol zur Behandlung der PPH 1995

E) Seit der 3. PPH-Weltkonferenz 2004 in Italien

II 5. Die PH ist aktuell definiert durch

A) den echokardiografischen Nachweis eines erhöhten systolischen pulmonalarteriellen Drucks.

B) einen per Rechtsherzkatheter gemessenen mittleren pulmonalarteriellen Druck $\geq 25$ $\mathrm{mmHg}$ in Ruhe.

C) ein mittels Rechtsherzkatheter gemessenes erniedrigtes Herzzeitvolumen.

D) einen invasiv gemessenen transpulmonalen Gradienten $\leq 12 \mathrm{mmHg}$

E) einen invasiv gemessenen transpulmonalen Gradienten $\geq 12 \mathrm{mmHg}$

II 6. Welches Diagnoseverfahren sollte bei Verdacht auf eine PH immer durchgeführt werden?

A) Linksherzkatheter

B) Pulmonale Angiografie

C) 24 -Stunden-Blutdruckregistrierung

D) 24 -Stunden-EKG

E) Echokardiografie

II 7. Welche Methode ist zum Ausschluss einer chronisch thromboembolischen PH am sensitivsten?

A) Die Echokardiografie mit Erfassung des

Lungenvenenflusses im linken Vorhof

B) Die Röntgen-Thorax-Untersuchung

C) Die thorakale CT mit Kontrastmittel

D) Die Magnetresonanztomografie

E) Die Ventilationsperfusionszintigrafie
II 8. Welche PH-Patienten können mit Kalziumkanal-Blockern behandelt werden?

A) Alle Patienten der funktionellen Klassen (FC) II und III.

B) Alle Patienten der FC I „Pulmonale Arterielle Hypertonie".

C) Alle Patienten mit hämodynamisch nachgewiesener Reagibilität, d. h. Responder.

D) Die Responder unter den Patienten mit idiopathischer pulmonaler arterieller Hypertonie.

E) Nur PAH-Patienten, die andere Therapien nicht vertragen.

\section{9. Eine Kombinationstherapie ist bei PAH} sinnvoll

A) bei allen Patienten.

B) bei Patienten, deren körperliche Leistungsfähigkeit sich unter einer Monotherapie nicht normalisiert.

C) nur bei den Patienten mit einem mittleren pulmonalarteriellen Druck > $50 \mathrm{mmHg}$.

D) nur bei den Patienten mit echokardiografischen Zeichen der Rechtsherzinsuffizienz.

E) bei Patienten, die sich unter Monotherapie nicht ausreichend stabilisieren und die therapeutischen Zielkriterien nicht erreichen.

\section{10. Wie sollte der Verlauf einer PH}

kontrolliert werden?

A) Unabhängig vom Befinden des Patienten alle sechs Monate

B) Unabhängig vom Zustand des Patienten alle drei Monate

C) Maximal einmal jährlich in einem $\mathrm{PH}$-Zentrum

D) In Absprache mit dem PH-Zentrum je nach individuellen Erfordernissen

E) Bei akuter Verschlechterung und nur durch den Hausarzt

\section{Fortbildungszertifikat}

Hier können Sie CME-Punkte sammeln, und zwar a) für die Pflichtfortbildung aller Vertragsärzte und b) für freiwillige Fortbildungszertifikate, die viele Landesärztekammern (z. B. Bayerische LÄK) anbieten.

Bei erfolgreicher Teilnahme (mindestens 70\% richtig beantwortete Fragen) vergibt die Bayerische Landesärztekammer, mit der die Zeitschrift CardioVasc kooperiert, 2 Punkte, bei richtiger Beantwortung aller Fragen 3 Punkte. Die Punkte werden online dem Punktekonto der erfolgreichen Teilnehmer gutgeschrieben. CardioVasc bietet Ihnen die Möglichkeit, innerhalb von fünf Jahren bis zu 120 Punkte zu erwerben.

Weitere Informationen erhalten Sie im Internet unter www.cardiovasc.de
CME-Herausgeber- und Review-Board: Prof. Dr. C. Diehm, Prof. Dr. J. Hoyer, Prof. Dr. G. Klose, Priv.-Doz. Dr. F. Masuhr, Prof. Dr. G. Nickenig, Prof. Dr. H. Schunkert, Prof. Dr. J. Seißler, Prof. Dr. D. Tschöpe, Prof. Dr. A. Weizel, Prof. Dr. A. Wirth, Prof. Dr. W. Zidek 